\title{
Pólipos y adenomas de la vesícula biliar: consideraciones clínico-patológicas
}

\author{
Iván Roa $\mathrm{E}^{1}$, Xabier de Aretxabala U ${ }^{2}$, René Morgan $\mathrm{F}^{\mathrm{a}}$, \\ Ricardo Molina $U^{a}$, Juan C Araya $0^{1}$, Juan Roa $\mathbf{S}^{1}$, \\ Gilda Ibacahe $\mathbf{S}^{1}$.
}

\section{Clinicopathological features of gallbladder polyps and adenomas}

Background: Chile has a high frequency of gallbladder cancer. Polyps are common lesions of gallbladder mucosa but there is little information about their morphological features. Aim: To report the clinical and pathological features of 219 gallbladder polyps. Material and methods: Cholecystectomies samples in which a polypoid lesion was diagnosed microscopically. In all cases, complete clinical information and digitalized images of the complete surgical specimens was reviewed. Results: In a period of 10 years, 21.412 gallbladders were processed. Among these, 884 carcinomas were diagnosed and in 219 cases (1\%) a polyp was found. One hundred and eighty three patients were females (mean age 49.3 years) and 36 males (mean age 53.4 years). The preoperative diagnosis of gallbladder polyp was done only in 26 cases (12\%). Eighty five percent of polyps were non-neoplastic (metaplastic in $32 \%$, cholesterol in $29 \%$, hyperplastic in $22 \%$ and inflammatory in $2 \%$ ). The remaining $15 \%$ were adenomas. Seventy five percent of non-neoplastic polyps were located in the proximal half of the gallbladder and $88 \%$ of adenomas in the distal half. Ninety five percent of non-neoplastic polyps measured less than $10 \mathrm{~mm}$. Among adenomas, 47\% measured less than $5 \mathrm{~mm}$ and $28 \%$ more than $10 \mathrm{~mm}$. Smaller polyps were of cholesterol and larger polyps were adenomas. Eight adenomas were associated with an adenocarcinoma, two had less than $5 \mathrm{~mm}$ lenght. Mean age of patients with adenomas associated to cancer was higher than patients with pure adenomas (64.6 and 44.3 years respectively, $\mathrm{p}>0.001$ ). Conclusions: There are size and location differences between non neoplastic polyps and adenomas. Adenomas associated to cancer may measure less than $5 \mathrm{~mm}$. Therefore the polyp size criteria to decide surgical behavior in symptomatic gallstone patients may be misleading (Rev Méd Chile 2004; 132: 673-9).

(Key Words: Biliary tract surgical procedures; Cholelithiasis; Gallbladder neoplasms)

Recibido el 27 de octubre, 2003. Aceptado en versión corregida el 1 de abril, 2004.

Trabajo financiado por Dirección de Investigación, Universidad de la Frontera \#140305.

${ }_{1}^{1}$ Departamento de Anatomía Patológica, Facultad de Medicina, Universidad de la Frontera.

${ }^{2}$ Departamento de Cirugía, Facultad de Medicina, Universidad de la Frontera.

anterno de la carrera de medicina

Correspondencia a: Dr. Iván Roa E. Casilla 54- D. Temuco,

Chile. E-mail: iroa@ufro.cl 
C hile presenta la mayor frecuencia de cáncer de la vesícula biliar del mundo, en ambos $\operatorname{sexos}^{1-3}$. En la mujer, representa la primera causa de muerte por tumores malignos desde 1985, con una proporción de 4:1 respecto de los hombres ${ }^{3,4}$. Los pólipos vesiculares son lesiones frecuentes en la mucosa vesicular, los que en su gran mayoría corresponden a lesiones de carácter reactivo, adaptativo o inflamatorio ${ }^{5,6}$. Sin embargo, también se expresan como lesiones polipoideas los tumores glandulares benignos o adenomas y los malignos 0 adenocarcinomas. La mayor parte de las lesiones polipoideas no adenomatosas, rara vez sobrepasan un centímetro y su seguimiento excepcionalmente muestra un crecimiento significativo ${ }^{7}$. Los adenomas, por su parte, pueden alcanzar tamaños mayores de $1 \mathrm{~cm}$. Los pólipos mayores de $1 \mathrm{~cm}$, solitarios, sésiles y ecodensos, tienen una mayor probabilidad de corresponder a adenomas, por lo cual tendrían indicación quirúrgica y aun cuando un pequeño porcentaje de ellos sufrirá transformación maligna a adenocarcinoma, hasta la fecha no es posible predecir cuál lo hará ${ }^{8-10}$. Por otro lado, el seguimiento de lesiones menores de $10 \mathrm{~mm}$, ha demostrado que, a lo menos un tercio de ellas desaparecen, siendo las restantes benignas ${ }^{11}$.

En el adenocarcinoma de la vesícula biliar, se reconocen dos grandes modelos a través de los cuales se produciría la transformación maligna: la secuencia displasia-carcinoma ${ }^{12-15}$ y la secuencia adenoma-carcinoma ${ }^{16,17}$. La primera es a partir de las alteraciones del epitelio propio de la mucosa vesicular, en el que aparece una metaplasia. Sobre esta metaplasia aparece la displasia, la cual progresa a carcinoma in situ y posteriormente a invasor. La segunda vía propone la transformación maligna a partir de un tumor glandular inicialmente benigno, como son los adenomas. Evidencia experimental y clínica sostienen a ambos modelos en la vesícula biliar, a nivel genético-molecular, se ha demostrado que ambas vías corresponden a dos eventos biológicos distintos ${ }^{12,18-20}$.

En consideración a la alta incidencia de la patología tumoral de la vesícula biliar en nuestro país y frente a la problemática que plantea el hallazgo de una lesión polipoidea en la mucosa vesicular, es que consideramos oportuno estudiar las características clínicas y morfológicas de estas lesiones.

\section{MATERIAL Y MÉTODO}

Selección de casos. Durante la década 19932002, en nuestra institución se procesaron histológicamente 21.412 piezas de colecistectomías. En ese mismo período, se diagnosticaron 884 cánceres de vesícula biliar $(4,1 \%)$. En 219 casos se estableció el diagnóstico microscópico de pólipo vesicular. En todos los casos se obtuvo la información clínica completa, además de las imágenes digitalizadas de las piezas quirúrgicas intactas, que permitieron un mejor y más detallado examen macroscópico, especialmente relacionado con la cantidad, tamaño, localización, distribución, asociación con otras lesiones, etc.

Protocolo de procesamiento. El procesamiento de las piezas quirúrgicas de colecistectomía fue realizado en forma uniforme, de acuerdo a protocolos establecidos desde hace más de una década ${ }^{21}$, el que resumidamente consiste en: apertura de la vesícula biliar, fijación y extensión de la pieza quirúrgica en planchas de parafina sólida, fotografía o digitalización de la pieza con un examen macroscópico diferido en búsqueda de lesiones sospechosas. Posteriormente, la toma de muestras representativas de cada uno de los segmentos para estudio histológico, o bien, el mapeo completo de la pieza quirúrgica en presencia de lesiones preneoplásicas o neoplásicas de la mucosa vesicular.

Clasificación histológica. Las lesiones polipoideas fueron diagnosticadas de acuerdo a criterios previamente reportados por otros autores ${ }^{14,22}$. Se reconocieron las lesiones neoplásicas malignas y los adenomas en sus distintas variedades, estos últimos por definición con algún grado de displasia. Los pólipos no neoplásicos $(\mathrm{PnN})$ se clasificaron como hiperplásicos, principalmente constituidos por mucosa propia con presencia de estructuras glandulares normotípicas frecuentemente dilatadas, rodeadas por estroma laxo histológica; los pólipos de carácter metaplásico, ya sea de tipo pilórico-antral y de tipo intestinal con células caliciformes. Además de los de colesterol, inflamatorios y otros de fácil identificación (Figura 1). 


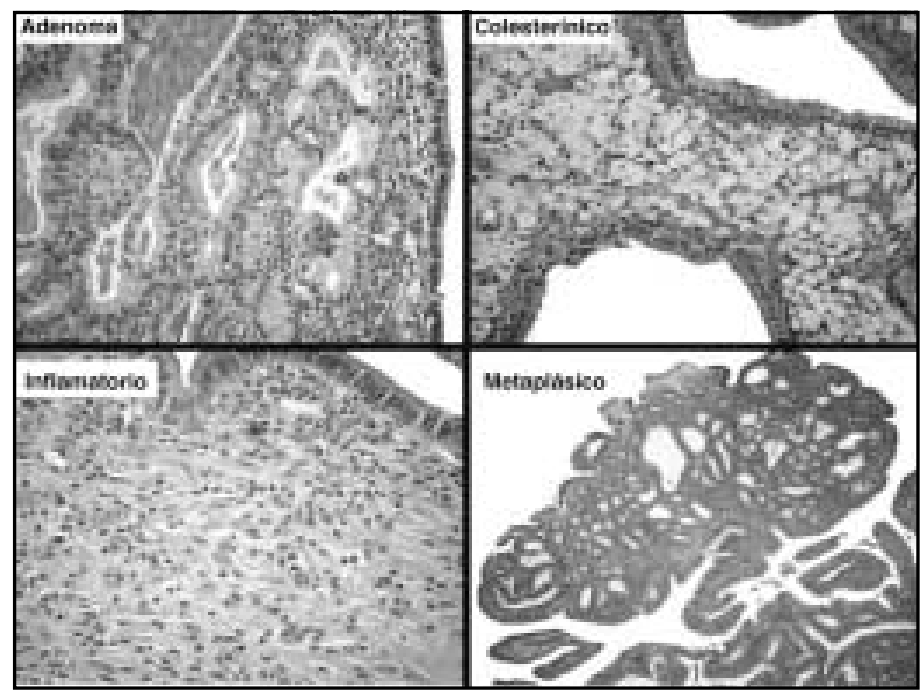

FiguRa 1. Morfología microscópica de pólipos vesiculares. Izquierda superior: adenoma tubular con displasia leve, caracterizado por proliferación de estructuras glandulares de tipo tubular con escaso estroma. Derecha superior: pólipo de colesterol con gran cantidad de histiocitos espumosos en el corión de la mucosa. Izquierda inferior: pólipo de tipo inflamatorio: ausencia de glándulas con un estroma de tejido granulatorio e infiltrado inflamatorio polimorfonuclear. Derecha inferior: Pólipo metaplásico: proliferación de estructuras tubulares y acinares dilatadas constituidas por células mucosas de tipo pilórico.

Estadística. Se realizó análisis de la varianza (ANOVA) y t-test para los promedios, chi cuadrado y test exacto de Fisher para tablas de contingencia. Se consideró como significativo a un $\mathrm{p}$ $<0,05$.

\section{RESULTADOs}

El grupo total incluyó 219 pólipos vesiculares, correspondientes a 183 mujeres (84\%) y 36 hombres (16\%) con un promedio de edad de 49,3 y 53,4 años, respectivamente. Los diagnósticos preoperatorios en $84 \%$ correspondieron a litiasis o inflamación vesicular aguda. En 26 casos (12\%) se sugirió la presencia de un pólipo vesicular, $25 \mathrm{de}$ ellos correspondieron a PnN y sólo un caso a adenoma. Los pólipos más frecuentes fueron los de tipo metaplásico en 69 casos (32\%) (Tabla 1). Le siguieron en frecuencia los de colesterol y los hiperplásicos. En 32 casos (15\%) correspondieron a adenomas, la mayor parte de ellos de tipo tubular. Un caso correspondió a pólipo hamartomatoso de tipo Peutz-Jegher en un niño de 2 años y en un caso la lesión correspondió a un foco de adenomiosis vesicular. Al igual que en el resto de la patología biliar, hubo predominio en las mujeres (85\%), sin embargo, en los adenomas este porcentaje fue levemente superior alcanzando a 91\% (29 de 32 casos).

Se observaron diferencias en los promedios de edad entre los distintos tipos de pólipos, sin

Tabla 1. C aracterísticas de 217 pólipos de la vesícula biliar

\begin{tabular}{|c|c|c|c|c|c|c|}
\hline & $\begin{array}{c}\text { Tipo } \\
\text { n }\end{array}$ & $\%$ & $\begin{array}{c}\text { Tamaño } \\
\text { Promedio } \\
\overline{\mathrm{x}}(\mathrm{Mm})\end{array}$ & DS & $\overline{\mathrm{x}}$ (años) & DS \\
\hline Metaplásico & 69 & 32 & 4,8 & 4 & 56 & 16 \\
\hline Colesterol & 64 & 29 & 3,0 & 2 & 42 & 13 \\
\hline Hiperplásico & 48 & 22 & 4,3 & 2,6 & 53 & 17 \\
\hline Inflamatorio & 4 & 2 & 4,5 & 2,6 & 48 & 4,6 \\
\hline Adenoma & 32 & 15 & 7,2 & 5,5 & 49 & 17 \\
\hline Total & 217 & 100 & 4,4 & 4,8 & 50 & 16 \\
\hline
\end{tabular}

Se excluyeron 2 casos: una adenomiosis y un pólipo hamartomatoso de tipo Peutz-Jegher. 
embargo, éstos no fueron significativos. Los pacientes con pólipos de colesterol fueron el grupo más joven (promedio 41,7 años), en cambio, los pacientes con pólipos metaplásicos e hiperplásicos fueron los de mayor edad (promedio 55,8 años y 53,1 años, respectivamente) (Tabla 1). Los pacientes con adenomas tuvieron en promedio 49,4 años, observándose una marcada diferencia entre mujeres y hombres (47,6 años y 66,6 años, respectivamente). Sólo tres hombres constituían este grupo, lo cual no permitió su análisis.

El 63\% de los pólipos, independiente de su naturaleza, eran únicos, $14 \%$ doble y $23 \%$ eran múltiples. El $62 \%$ de los PnN y el $69 \%$ de los adenomas eran únicos. El 31\% de los adenomas eran dobles o múltiples, similar a los PnN (38\%), observándose en $24 \%$ y $18 \%$ múltiples pólipos (más de tres) en ambos grupos.

Respecto de la localización, 71\% de los pólipos estaban en los dos tercios proximales (cuerpo y cuello vesicular) y en menor proporción en el fondo vesicular. El $75 \%$ de los PnN estaban localizados en la mitad proximal de la vesícula biliar, en cambio, los adenomas en $88 \%$ estaban en la mitad distal $(p=0,006)$.

En 152 casos (69\%) las lesiones medían menos de $5 \mathrm{~mm}, 91 \%$ medía menos de $10 \mathrm{~mm}$ de diámetro y sólo 8 lesiones medían más de $15 \mathrm{~mm}$. El 95\% de los PnN medía menos de $10 \mathrm{~mm}$, en cambio, $47 \%$ de los adenomas medía menos de 5 $\mathrm{mm}$ y sólo $28 \%$ de ellos medían más de $1 \mathrm{~cm}$. El tamaño promedio de los pólipos fue de $4,4 \mathrm{~mm}$ (Tabla 1). Los de menor tamaño fueron los de colesterol, los que alcanzaron $3 \mathrm{~mm}$ en promedio, en cambio, los de mayor tamaño fueron los adenomas con 7,2 mm de promedio. La diferencia de tamaño entre los PnN y los adenomas fue significativa ( $p<0,00001$ ). El 96\% de los adenomas puros eran de tipo tubular con diversos grados de displasia, en cambio, de los 8 adenomas asociados a un cáncer vesicular, la mitad de ellos presentaron un patrón de tipo papilar o túbulo-papilar.

Los adenomas que se asociaron a la presencia de un adenocarcinoma vesicular, en cinco de 8 casos estaban en el fondo vesicular y los tres restantes en el cuerpo, seis de ellos eran únicos. En cinco casos los adenomas median más de 10 $\mathrm{mm}$ y dos casos medían menos de $5 \mathrm{~mm}$ de diámetro, ambos fueron carcinomas incipientes (mucosos). Los promedios de edad de los pacientes con adenomas asociados a un cáncer fueron significativamente mayores que los adenomas puros $(64,6$ años versus 44,3 años, $p=0,0001)$. Aun cuando los adenomas asociados a un cáncer fueron en promedio de mayor tamaño $(7,2 \mathrm{~mm}$ versus $6,4 \mathrm{~mm})$, estas diferencias no fueron significativas. El nivel de infiltración de los cánceres asociados a adenomas en cuatro casos eran mucosos, tres musculares y sólo un caso con microinfiltración de la subserosa. Este último caso correspondió a adenomas múltiples, el mayor de los cuales medía $12 \mathrm{~mm}$.

\section{DisCUSIÓN}

Nuestros resultados corresponden a la mayor serie de lesiones polipoideas de la vesícula biliar comunicada en nuestro país, siendo similares a los observados por otros autores nacionales ${ }^{11,23,24}$.

Se confirma la relación entre el tamaño del pólipo y su naturaleza (los no neoplásicos son significativamente más pequeños), sin embargo, alrededor de $50 \%$ de los adenomas son lesiones pequeñas menores de $5 \mathrm{~mm}$ y los dos adenomas asociados a cáncer medían menos de $5 \mathrm{~mm}$. En pacientes con litiasis sintomática, la presencia de un pólipo vesicular le agrega un factor más a la indicación quirúrgica. Aun cuando la gran mayoría de estas lesiones corresponderán a lesiones no tumorales, la colecistectomía está indicada por la litiasis más que por el pólipo ${ }^{8,24,25}$. En cambio, los sujetos portadores de una litiasis asintomática y pólipo vesicular, la indicación quirúrgica debería considerarse en relación a la edad, sexo, factores de riesgo de cáncer vesicular y características del pólipo como tamaño, localización y características radiológicas o ultrasonográficas ${ }^{5,26,27}$. El hallazgo de 2 adenomas menores de $5 \mathrm{~mm}$ asociados a carcinoma en esta serie, plantean dudas respecto del seguimiento de estos pacientes a través de imágenes. Los exámenes de imágenes de la mucosa vesicular en presencia de cálculos no son todo lo sensible y específicos que pudiésemos esperar ${ }^{28-30}$. Hay trabajos que demuestran un alto grado de error en la evaluación de este tipo de lesiones, lo que pudiese inducir a una decisión errónea ${ }^{29,31}$. 
Un hecho destacable es que, a diferencia de lo reportado en otras series, los pólipos de colesterol no son los más frecuentes en el material de colecistectomías y sólo representan el $29 \%$ de nuestros casos. Esta cifra guarda estrecha relación con la frecuencia de colesterolosis que hemos observado en las colecistectomías (33\%). Existe muy escasa información en la literatura acerca de lesiones polipoideas vesiculares en pacientes sin cálculos ${ }^{32,33}$. En este grupo de pacientes, la colesterolosis vesicular pudiese ser la principal causa de pólipos ${ }^{34}$, debiendo considerar que los pólipos colesterínicos se observan en los sujetos más jóvenes y el 73\% de los PnN miden menos de $5 \mathrm{~mm}$. El tamaño límite propuesto para un pólipo vesicular benigno sería de $1 \mathrm{~cm}^{8,17,35}$, estos elementos podrían sugerir una conducta contemplativa frente a un pólipo vesicular en sujetos sin litiasis, sin embargo, el seguimiento periódico sería mandatario ${ }^{24,36}$. Los carcinomas asociados a adenomas en 7 de los 8 casos fueron lesiones incipientes, demostrándose infiltración de la subserosa sólo en un caso.

En nuestros casos los adenomas se localizaron en la mitad distal de la vesícula biliar a diferencia de los PnN que se encontraron preferentemente en la mitad proximal (cuello) de la vesícula biliar.

Respecto de la importancia biológica de los adenomas como lesiones precursoras de un cáncer vesicular, encontramos 8 casos de cánceres asociados a adenomas, lo que representa una frecuencia de 0,9\% del total de cánceres que hemos diagnosticado en igual período. Esto traduciría su escasa importancia como lesiones precursoras del cáncer vesicular. En un estudio

\section{REFERENCIAS}

1. Lazcano-Ponce EC, Miquel JF, Muñoz N, Herrero R, FerRecio C, Wistuba II et al. Epidemiology and molecular pathology of gallbladder cancer. CA Cancer J Clin 2001; 51: 349-64.

2. Nervi F. Cáncer de la vesícula biliar en Chile. Rev Méd Chile 2001; 129: 979-81.

3. Medina E, KaEMPFFER AM. Mortalidad por cáncer en Chile: Consideraciones epidemiológicas. Rev Méd Chil 2001; 129: 1195-202. de 196 cánceres vesiculares incipientes, no observamos focos adenomatosos adyacentes al tumor, a excepción de $4 \operatorname{casos}^{37}$. La vía carcinogénica adenoma-carcinoma, si bien existe en la vesícula biliar, su importancia es significativamente menor a la demostrada en otros tumores del tubo digestivo, como en el cáncer colorrectal o en el carcinoma ampular, donde residuos adenomatosos se observan hasta en 32\% y $92 \%$ de los casos respectivamente ${ }^{38,39}$. La evidencia a nivel molecular también avala la existencia de estas dos vías carcinogénicas, siendo la secuencia displasia-carcinoma la más aceptada para el carcinoma vesicular ${ }^{19,40,41}$. La secuencia carcinogénica adenoma-carcinoma en la vesícula biliar fue sugerida por Kozuka ${ }^{17}$, quien reportó una alta frecuencia de adenomas y residuos adenomatosos en los cánceres vesiculares, cifras que no han podido ser reproducidas. Las diferencias pudiesen ser explicadas en base a los criterios histológicos empleados en el diagnóstico de las lesiones displásicas y adenomas por los autores japoneses respecto de los patólogos occidentales, controversia aún más marcada en órganos como colon ${ }^{42,43}$ y estómago ${ }^{44}$.

En resumen, podemos señalar que los pólipos vesiculares son lesiones poco frecuentes (alrededor de $1 \%$ de las colecistectomías por litiasis sintomática) y que los pólipos no tumorales presentan significativas diferencias en tamaño y localización respecto de los adenomas. Los adenomas asociados a cáncer pueden ser menores de 10 $\mathrm{mm}$ y la secuencia adenoma-carcinoma en la vesícula biliar no parece tener un rol de importancia en la carcinogénesis vesicular.

4. SERRA I. ¿Ha disminuido el cáncer de la vesícula biliar en Chile? Rev Méd Chile 2001; 129: 1079-84.

5. Myers RP, ShafFer EA, Beck PL. Gallbladder polyps: epidemiology, natural history and management. Can J Gastroenterol 2002; 16: 187-94.

6. Boulton RA, Adams DH. Gallbladder polyps: when to wait and when to act. Lancet 1997; 349: 817.

7. Albores-Saavedra J, Vardaman CJ, Vuttch F. Nonneoplastic polypoid lesions and adenomas of the gallbladder. Pathol Annu 1993; 28 Pt 1: 145-77. 
8. Terzi C, Sokmen S, Seckin S, Albayrak L, Ugurlu M. Polypoid lesions of the gallbladder: report of 100 reference to operative indications. Surgery 2000; 127: 622-7.

9. Mainprize KS, Gould SW, Gilbert JM. Surgical management of polypoid lesions of the gallbladder. Br J Surg 2000; 87: 414-7.

10. Furukawa H, Kosuge T, Shimada K, Yamamoto J, KanaI Y, MuKaI K et aL. Small polypoid lesions of the gallbladder: surgical indications by helical computed tomography. Arch Surg 1998; 133: 7359.

11. Csendes A, Burgos AM, Csendes P, Smok G, Rojas J. Late follow-up of polypoid lesions of the gallbladder. Ann Surg 2001; 234: 657-60.

12. Kim YT, Kim J, Jang YH, Lee WJ, Ryu JK, Park YK et AL. Genetic alterations in gallbladder adenoma, dysplasia. Cancer Lett 2001; 169: 59-68.

13. Roa I, Araya JC, Villaseca M, De Aretxabala X, Ruedemann P, ENDoh K et al. Preneoplastic lesions and gallbladder cancer: an estimate of the period required for progression. Gastroenterology 1996; 111: 232-6.

14. Albores-Saavedra J, Henson DE, Sobin LH. The WHO Histological Classification of Tumors of the Extrahepatic Bile Ducts. A commentary on the second. Cancer 1992; 70: 410-4.

15. LAITIO M. Histogenesis of epithelial neoplasms of human gallbladder I. Dysplasia. Pathol Res Pract 1983; 178: 51-6.

16. Nakajo S, Yamamoto M, Tahara E. Morphometrical analysis of gallbladder adenoma and adenocarcinoma with reference to histogenesis and adenoma-carcinoma sequence. Virchows Arch A Pathol Anat Histopathol 1990; 417: 49-56.

17. Kozuka S, Tsubone N, Yasui A, Hachisuka K. Relation of adenoma to carcinoma in the gallbladder. Cancer 1982; 50: 2226-34.

18. Wistuba, II, Albores-SaAvedra J. Genetic abnormalities involved in the pathogenesis of gallbladder carcinoma. J Hepatobiliary Pancreat Surg 1999; 6: 237-44.

19. Chang HJ, Jee CD, Kim WH. Mutation and altered expression of beta-catenin during gallbladder carcinogenesis. Am J Surg Pathol 2002; 26: 75866.

20. Wistuba II, Miquel JF, Gazdar AF, Albores-Saavedra J. Gallbladder adenomas have molecular abnormalities different from those present in gallbladder carcinomas. Hum Pathol 1999; 30: 21-5.
21. Roa I, Araya JC, Wistuba I, de AretXabala X. Cáncer de la vesícula biliar: consideraciones anatómicas y anátomo-patológicas. Rev Méd Chile 1990; 118: 572-9.

22. Yamamoto M, NaKajo S, Tahara E. Histological classification of epithelial polypoid gallbladder. Acta Pathol Jpn 1988; 38: 181-92.

23. Smok G, BentJerodt R, Csendes A. Lesiones polipoideas benignas de la vesícula biliar y su relación con el adenocarcinoma. Rev Méd Chile 1992; 120: 31-5.

24. Jiron MI, Silva H, Whittle C, Fuster F, Madrid AM, Brahm J et al. Pólipos vesiculares. Segundo Taller de la Sociedad de Hepatología de Chile. Rev Méd Chile 1994; 122: 1316-7.

25. Chijina K, TanaKa M. Polypoid lesion of the gallbladder: indications of after surgery for malignant polypoid lesion. Int Surg 1994; 79: 106-9.

26. Moriguchi $H$, Tazawa J, Hayashi $Y$, Takenawa $H$, NAKAYAMa E, Marumo F ET aL. Natural history of polypoid lesions in the gallbladder. Gut 1996; 39: 860-2.

27. Patino JF, Quintero GA. Asymptomatic cholelithiasis revisited. World J Surg 1998; 22: 1119-24.

28. Azuma $T$, Yoshikawa $T$, Araida $T$, TaKasaki $K$. Differential diagnosis of polypoid lesions of the gallbladder by endoscopic ultrasonography. Am J Surg 2001; 181: 65-70.

29. Damore LJ 2ND, Cook CH, Fernández KL, CunningHAM J, Euson EC, MeLvin WS. Ultrasonography incorrectly diagnoses gallbladder polyps. Surg Laparosc Endosc Percutan Tech 2001; 11: 88-91.

30. Furukawa $H$, takayasu K, Mukai K, Inoue K, Kyokane T, Shimada K ET aL. CT evaluation of small polypoid lesions of the gallbladder. Hepatogastroenterology 1995; 42: 800-10.

31. Muguruma N, Okamura S, Ichikawa S, Tsujigami K, SuZUKi M, TADATSU M ET al. Endoscopic sonography in the diagnosis of gallbladder patients with gallstones. J Clin Ultrasound 2001; 29: 395-400.

32. Jones-Monahan KS, Gruenberg JC, Finger JE, Tong GK. Isolated small gallbladder polyps: an indication for symptomatic patients. Am Surg 2000; 66: 716-9.

33. Tinsiey AR, Mulkerin LE, VAn der Linde JM, Todd DW. Polypoid lesions of the acalculous gallbladder. South Med J 1975; 68: 958-62.

34. MarTínEZ G DLRJ. Neoplasias y displasias de vesícula biliar y su relación con litiasis. Estudio clinicopatológico de casos y controles. Rev Gastroenterol Mex 1998; 63: 82-8. 
35. Horiguchi Y. How to determine the surgical indications for lesions of the gallbladder? J Gastroenterol 2001; 36: 648-9.

36. Csendes A, Burgos AM, Csendes P, Smok G, Rojas J. Late follow-up of polypoid lesions of the gallbladder smaller than $10 \mathrm{~mm}$. Ann Surg 2001; 234: 657-60.

37. Roa I, de Aretxabala X, Araya JC, Viliaseca M, Roa J, GuZmán P. Carcinoma incipiente de la vesícula biliar. Estudio clínico-patológico y pronóstico en 196 casos. Rev Méd Chile 2001; 129: 1113-20.

38. Chen CD, Yen MF, Wang WM, Wong JM, Chen TH. A case-cohort study for the disease natural history and de novo carcinoma and surveillance of colon and polypectomy: implication for efficacy of colonoscopy. Br J Cancer 2003; 88: 1866-73.

39. Kaiser A, Jurowich C, Schonekas H, Gebhardt C, Wunsch $\mathrm{PH}$. The adenoma-carcinoma sequence applies to epithelial papilla of Vater. Z Gastroenterol 2002; 40: 913-20.
40. Rashid A, Gao YT, Bhakta S, Shen MC, Wang BS, DENG J ET AL. Beta-catenin mutations in biliary tract cancers: a population-based study in China. Cancer Res 2001; 61: 3406-9.

41. Yanagisawa N, Mikami T, Saegusa M, Okayasu I. More frequent beta-catenin exon 3 mutations in gallbladder adenomas than in carcinomas indicate different lineages. Cancer Res 2001; 61: 19-22.

42. JASS JR. Histopathology of early colorectal cancer. World J Surg 2000; 24: 1016-21.

43. Schlemper RJ, Itabashi M, Kato Y, Lewin KJ, Riddell $\mathrm{RH}$, Shimoda T ET AL. Differences in the diagnostic criteria used by pathologists to diagnose colorectal carcinoma. Cancer 1998; 82: 60-9.

44. Schlemper RJ, Kato Y, Stolte M. Diagnostic criteria for gastrointestinal carcinomas countries: proposal for a new classification system epithelial neoplasia. J Gastroenterol Hepatol 2000; 15 Suppl: G49-57. 